\title{
Avaliação do edema macular após cirurgia não complicada de facoemulsificação com implante de lente intraocular por meio da tomografia de coerência óptica spectral domain
}

\author{
Evaluation of macular edema after uncomplicated phacoemulsification surgery with implantation \\ of intraocular lens by spectral domain optical coherence tomography
}

Everton Paroschi Corrếa ${ }^{1}$, Luciana Freitas lemos de Oliveira ${ }^{1}$, Pedro Durães Serracarbassa ${ }^{2}$, Aky ioshi Oshima ${ }^{1}$, Emerson Fernandes de Sousa e Castro ${ }^{3}$

\section{RESUMO}

Objetivo: Avaliar o edema macular após cirurgia não complicada de facoemulsificação com implante de lente intraocular por meio da tomografia de coerência óptica spectral domain (OCT-SD).

Métodos: Foi realizado estudo prospectivo em 62 olhos de pacientes submetidos à facoemulsificação com implante de lente intraocular. Os pacientes foram avaliados antes e após a cirurgia, no primeiro dia, e na primeira, segunda e quarta semanas. Acuidade visual (AV), células na câmara anterior e tomografia de coerência óptica macular cubo 200×200 foram medidos.

Resultados: Após a cirurgia de facoemulsificação houve melhora da acuidade visual, inflamação decrescente, e a espessura e o volume macular aumentaram. Ocorreu um caso de edema macular cistoide. Houve correlação inversa fraca entre a acuidade visual e a espessura macular central, e entre a acuidade visual e o volume macular. Foi observada correlação direta fraca entre a inflamação e o volume macular. Conclusão: Edema macular subclínico desenvolve-se mesmo após cirurgia de catarata não complicada em pacientes não predispostos. A tomografia de coerência óptica spectral domain foi capaz de detectar pequenos aumentos na espessura macular no período avaliado.

Descritores: Facoemulsificação; Edema macular; Tomografia de coerência óptica; Implante de lente intraocular; Período pós-operatório; Acuidade visual

\section{ABSTRACT}

Purpose: To evaluate macular edema after uncomplicated phacoemulsification with implantation of intraocular lens by spectral domain optical coherence tomography (OCT)

Methods: Prospective study was conducted in 62 eyes of patients underwent phacoemulsification with implantation of intraocular lens. Patients were evaluated before surgery and after surgery at day 1, week 1, week 2 and week 4. Visual acuity (VA), anterior chamber cells and 200x200 macular cube optical coherence tomography were measured.

Results: After phacoemulsification there was an improvement in visual acuity, decreasing inflammation, and increased macular thickness and macular volume. There was one case of cystoid macular edema. There was a weak inverse correlation between visual acuity and central macular thickness, and between visual acuity and macular volume. We observed a week direct correlation between inflammation and macular volume.

Conclusion: Subclinical macular edema develops even after uncomplicated cataract surgery in patients not predisposed. The spectral domain optical coherence tomography was able to detect small increases in macular thickness in the study period.

Keywords: Phacoemulsification; Macularedema; Tomography, optical coherence; Lens implantation, intraocular; Postoperative period; Visual acuity

\section{INTRODUÇÃO}

Edema macular (EM) permanece sendo a causa mais frequente de baixa visão após cirurgia de catarata ${ }^{(1,2)}$. O EM desenvolve-se devido à quebra da barreira hematorretiniana causando acúmulo de líquido no espaço extracelular da retina e formação de cistos nas camadas plexiforme externa e nuclear interna ${ }^{(3,4)}$.

A incidência de EM à angiografia (3-70\%) e de EM clinicamente relevante (0-22\%) variam substancialmente na literatura, dependendo da sua definição, técnica da cirurgia, método diagnóstico utilizado e comorbidades associadas $s^{(1,2,5-7)}$.

A incidência de EM pós-operatório aumenta quando a cirurgia de catarata é acompanhada no intraoperatório de rotura de cápsula posterior e, principalmente, por perda vítrea ${ }^{(8,9)}$. Entretanto, a incidência de EM subclínico, ou seja, aquele não detectado ao exame de biomicroscopia de fundo e sem prejuízo visual, após cirurgia de facoemulsificação sem complicações e resultados a longo prazo desta condição são menos claros.

A tomografia de coerência óptica (OCT) oferece várias vantagens sobre a angiografia fluoresceínica em termos de segurança, facilidade de uso e conforto para o paciente. Além disso, as análises quantitativas que o OCT proporciona permitem uma eficiente avaliação da progressão da doença e eficácia do tratamento(10). A angiografia, por sua vez, tem seu valor na demonstração dinâmica do comportamento vascular, podendo demonstrar vazamentos e outras alterações.
Submetido para publicação: 23 de outubro de 2012

Aceito para publicação: 1 de agosto de 2013

Trabalho realizado no serviço de Oftalmologia do Hospital do Servidor Público Estadual de São Paulo - HSPE/IAMSPE.

Médico, Departamento de Oftalmologia, Hospital do Servidor Público Estadual de São Paulo IAMSPE - São Paulo (SP), Brasil.

2 Médico, Departamento de Oftalmologia, Hospital do Servidor Público Estadual de São Paulo IAMSPE - São Paulo (SP), Brasil; Departamento de Oftalmologia do Hospital das Clínicas, Faculdade de Medicina, Universidade de São Paulo - São Paulo (SP), Brasil.

Médico, Departamento de Oftalmologia, Hospital do Servidor Público Estadual de São Paulo -

IAMSPE - São Paulo (SP), Brasil. Fundação Faculdade de Medicina, Universidade de São Paulo São Paulo (SP), Brasil.
Financiamento: Não houve financiamento para este trabalho.

Divulgação de potenciais conflitos de interesse: E.P.Corrêa, Nenhum; L.F.L.Oliveira, Nenhum; P.D.Serracarbassa, Nenhum; A.Oshima, Nenhum; E.F.S.Castro, Nenhum.

Endereço de correspondência: Everton Paroschi Corrêa. Av. Doutor Guilherme Dumont Villares, 2.000 - Apto. 12 - São Paulo (SP) - 05640-003 - Brasil

E-mail: evertonparoschi@yahoo.com.br 
O novo método de detecção da tomografia de coerência óptica spectral domain (OCT-SD) que utiliza um espectrômetro é mais eficiente, permitindo o aumento da sensibilidade quando comparado a sistemas equivalentes de OCT time domain ${ }^{(11-14)}$. A maior sensibilidade permite aquisições mais rápidas e de sinais mais fracos ${ }^{(15)}$. Dessa forma, resolução axial de cerca de $2 \mu \mathrm{m}$ é possível com OCT-SD(16). Imagens simples com 1000 A-scans podem ser captadas em 34,1 ms ou 1/29 de segundo. Esta velocidade de aquisição mais rápida permite a varredura de grandes áreas da retina.

Nesse estudo, utilizamos a tomografia de coerência óptica spectral domain para comparação das alterações qualitativas e quantitativas da mácula decorrentes da cirurgia de catarata. Esta tecnologia ainda não havia sido utilizada em nenhum estudo na pesquisa do edema macular após facoemulsificação, e esta é justamente a inovação do presente estudo.

\section{MÉTODOS}

Foram incluídos no estudo 65 olhos de 47 pacientes, que realizaram facectomia com implante de lente intraocular, e que se enquadraram nos critérios de inclusão. Foi obtida a assinatura do Termo de Consentimento Livre e Esclarecido. O presente estudo foi submetido à apreciação do Comitê de Ética em Pesquisa do Hospital dos Servidores Públicos Estaduais, sendo liberado sem restrições sob o número 097/09 junto ao CEP/IAMSPE.

\section{CRitérios de inclusão}

- Catarata de baixa densidade que permita a obtenção de uma imagem satisfatória no OCT pré-operatório;

- OCT com nota do exame maior ou igual a 7;

- Idade maior que 40 anos;

- Termo de Consentimento Livre e Esclarecido assinado;

- Pacientes que desejam e estão aptos a realizar as visitas de retorno durante o período do estudo.

\section{Critérios de excLusão}

- Qualquer retinopatia que proporcione baixa na acuidade visual;

- Diabete confirmada;

- História de uveíte;

- Glaucoma;

- Uso crônico de colírio, exceto lubrificantes;

- Uso crônico de corticoide sistêmico;

- História de trauma ocular;

- Realização de cirurgia ocular nos últimos 30 dias;

- Perda vítrea durante a cirurgia devido à rotura ou desinserção do saco capsular;

- Participação em qualquer outro estudo clínico de qualquer natureza nos últimos 180 dias.

Cada paciente foi submetido a exame oftalmológico no préoperatório, incluindo biomicroscopia anterior, biomicroscopia de fundo com lente de 78D e medida da melhor acuidade visual por meio da tabela de optotipos de Snellen, sempre na mesma sala e com a distância de 6 metros, além da tomografia de coerência óptica (OCT-Cirrus ${ }^{\circledR}$ Zeiss ${ }^{\circledR}$ ) da área macular. Foi utilizada a nota da intensidade de sinal conferida pelo próprio aparelho de OCT durante a realização dos exames, a qual pode variar de 0 a 10, e foi descartado todo exame com nota igual ou inferior a 6 . Foi preenchida uma ficha para cada paciente.

As primeiras 16 cirurgias foram realizadas pelo segundo autor, e as demais cirurgias foram realizadas pelo primeiro autor, sendo ambos residentes de terceiro ano com experiência semelhante. $\mathrm{O}$ exame de OCT e as avaliações pré e pós-operatórias foram feitas separadamente por cada cirurgião em cada um dos seus pacientes operados. O estudo foi realizado entre os meses de setembro de 2009 e novembro de 2010.
As cirurgias foram realizadas com aparelho Infiniti e caneta Ozil, implantada lente intraocular dobrável, sutura da incisão com nylon 10-0, injeção subconjuntival de gentamicina e dexametasona e curativo oclusivo. A anestesia utilizada foi bloqueio peribulbar com ou sem sedação.

Três olhos foram excluídos do estudo devido a complicações cirúrgicas (perda vítrea por desinserção ou rotura do saco capsular); portanto, 62 olhos foram incluídos nas análises estatísticas de 44 pacientes (foram utilizados os dados de um olho de 26 pacientes e dos dois olhos de 18 pacientes).

Foram realizadas consultas no primeiro dia, sete dias, 15 dias e um mês após a cirurgia com exame oftalmológico. Foi realizada medida da acuidade visual com e sem buraco estenopeico nos dias um, sete e 15. Foi aferida a acuidade visual com a melhor correção com um mês de pós-operatório. No 15 dia pós-operatório foi retirado o ponto da incisão principal. No sétimo e 30 dias foram feitos exames de OCT macular. Foi usado colírio de associação de moxifloxacino com dexametasona 2/2h por uma semana, seguido de colírio de dexametasona $1 \%$ em esquema de regressão por um mês.

A medida da inflamação ocular foi quantificada "em cruzes" de acordo com a classificação de células na câmara anterior segundo Standartization of Uveitis Nomenclature (SUN) Working Group, conforme observado na lâmpada de fenda.

Para avaliação dos resultados, as medidas de visão foram convertidas de Snellen para LogMAR.

Todos os exames de OCT foram feitos através de midríase farmacológica com tropicamida $1 \%$ colírio, três vezes a cada 5 minutos. Em cada exame foi realizado cubo 200×200 e"HD 5-line raster" da região macular. O OCT cubo 200x200 corresponde a um corte em cubo com definição de 200 linhas na horizontal e 200 linhas na vertical, já o "HD 5-line raster" corresponde a 5 linhas de alta resolução dispostos horizontalmente.

Os dados da espessura macular central, dos três milímetros perifoveais dos quatro quadrantes e do volume macular, obtidos através do OCT cubo 200×200, juntamente com os dados do exame clínico de cada paciente durante os diferentes momentos foram compilados e submetidos a análises estatísticas. O "OCT HD 5-line raster" (Anexo 5) foi utilizado para avaliação morfológica da mácula e, portanto, não entrou para os cálculos estatísticos.

Para responder o objetivo do estudo, primeiramente, foi realizado um teste de normalidade (Kolmogorov-Smirnov) das medidas avaliadas, sendo que todas as medidas de espessura e volume apresentaram normalidade de distribuição ( $p>0,05)$; já a acuidade visual e a medida de inflamação não apresentam normalidade de distribuição $(p<0,05)$. Foram descritas as medidas avaliadas segundo os momentos com uso de medidas resumo (média, desvio padrão, mediana, mínimo e máximo), e foram comparadas entre os momentos com uso de análises de variâncias com medidas repetidas, seguidas de comparações múltiplas de Bonferroni, para verificar entre quais momentos existem as diferenças nas medidas. A acuidade visual e a medida de inflamação foram comparadas entre os momentos com uso de teste de Friedman, seguido de comparações múltiplas não paramétricas para medidas repetidas.

Para avaliar a relação entre a AV e a espessura central e o volume, e entre a inflamação e a espessura central e o volume, foram calculadas as correlações de Spearman. As correlações foram calculadas juntando todos os momentos e em separado.

Os resultados foram ilustrados com uso de gráficos de perfis médios com os respectivos erros padrões e para acuidade visual (AV) e inflamação foram criados gráficos de barras com os valores medianos. Foram criados diagramas de dispersão para observar a relação entre AV e espessura central e entre inflamação e volume.

A análise estatística foi realizada em dois momentos distintos, sendo a primeira com os resultados dos primeiros 16 olhos, realizadas pelo primeiro cirurgião, e a segunda incluindo os resultados de todos os olhos ao final do estudo.

Os testes foram realizados com nível de significância de 5\%. 


\section{RESULTADOS}

Para elaboração deste estudo foram observados 62 olhos de 44 pacientes que realizaram a cirurgia de catarata, sendo que $63,6 \%$ foram do sexo feminino, e 36,4 do sexo masculino. A idade média foi de 71,64 anos com desvio padrão de 7,72 anos, sendo a idade mínima de 55 anos e máxima de 89 anos. A mediana foi de 71,50 anos.

Não houve diferença estatisticamente significante quando foram comparados os resultados obtidos pelos dois cirurgiões, o que mostra que os pacientes apresentaram espessura macular, volume macular, acuidade visual e inflamação similares após a cirurgia, não importando quem foi o cirurgião. Os gráficos e as tabelas incluídas no presente estudo foram confeccionados a partir do total de olhos avaliados.

Os gráficos 1 a 3 mostram que todas as medidas avaliadas se alteram ao longo dos momentos estudados, sendo que a medida da acuidade visual, em logMAR, diminui, o que demonstra a melhora na acuidade visual. Já a espessura macular e o volume macular aumentam com o tempo. Foi realizado o perfil médio da espessura macular nos quadrantes central, superior, inferior, nasal e temporal com seus respectivos erros padrões segundo os momentos e foi observado em todos o mesmo padrão apresentado no gráfico 2 (espessura central). A curva da espessura macular, em cada quadrante, sofre um aumento de menor intensidade na primeira semana, que se acentua em um mês. O volume macular sofre um aumento mais contínuo, porém com leve inclinação da curva entre uma semana e um mês. Foi observado melhora na acuidade visual de todos os pacientes, apesar do aumento da espessura macular. A única exceção foi um paciente que apresentou edema macular cistoide, um mês após a cirurgia, com piora da acuidade visual.

Na análise qualitativa macular, realizada pelo OCT, não foi evidenciada a presença de cistos ou líquido subfoveal em nenhum paciente, exceto no caso do paciente com edema macular cistoide. Este apresentou resolução completa das alterações anatômicas na mácula após três meses de seguimento.

No presente estudo foram avaliadas a medida da acuidade visual em logMAR, a espessura macular central, superior, inferior, nasal e temporal, o volume macular e a inflamação ocular. A tabela 1 mostra que todas as medidas avaliadas sofrem alteração estatisticamente significante ao longo dos momentos $(p<0,05)$, sendo esta a análise de variâncias de medidas repetidas ao longo de todos os momentos. Na avaliação prévia à cirurgia, o exame de OCT não captou adequadamente os valores da espessura temporal e do volume de um paciente, apesar de apresentar uma nota satisfatória dada pelo próprio aparelho, e portanto estas duas medidas não entraram para a análise estatística. Por isto estas duas medidas aparecem com o N de 61.

A inflamação ocular foi observada mais abundantemente somente no primeiro dia do pós-operatório. Na avaliação com sete dias de operados, houve poucos casos com inflamação residual, a qual desapareceu nos retornos posteriores, permanecendo apenas, após um mês, no caso do paciente com edema macular cistoide.

A tabela 2 mostra comparações múltiplas para cada medida entre os diferentes momentos dois a dois (pré-1 semana, pré-1 mês e 1 semana-1 mês), e para a inflamação (1 dia-1 semana, 1 dia-1 mês e 1 semana-1 mês). Foi observada que a medida da acuidade visual, o volume macular e a inflamação sofrem alteração estatisticamente significante entre todos os momentos, sendo que o valor da medida da acuidade visual e da inflamação diminui a cada momento avaliado e o valor do volume macular aumenta a cada momento $(p<0,05)$. A espessura macular central, e nos outros quatro quadrantes, não aumentou com valor estatisticamente significante na comparação pré1 semana $(p>0,05)$, havendo diferença significante nas comparações pré-1 mês e 1 semana-1 mês após a cirurgia $(p<0,05)$.

O gráfico 4 mostra um diagrama de dispersão entre a medida da acuidade visual e a espessura central, onde foi incluído apenas os

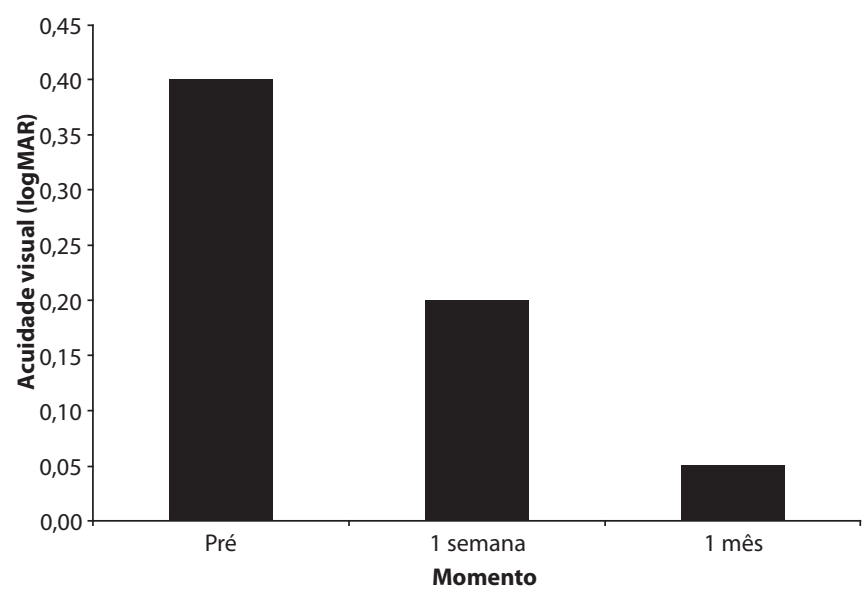

Gráfico 1. Valores medianos da medida da acuidade visual segundo os momentos.

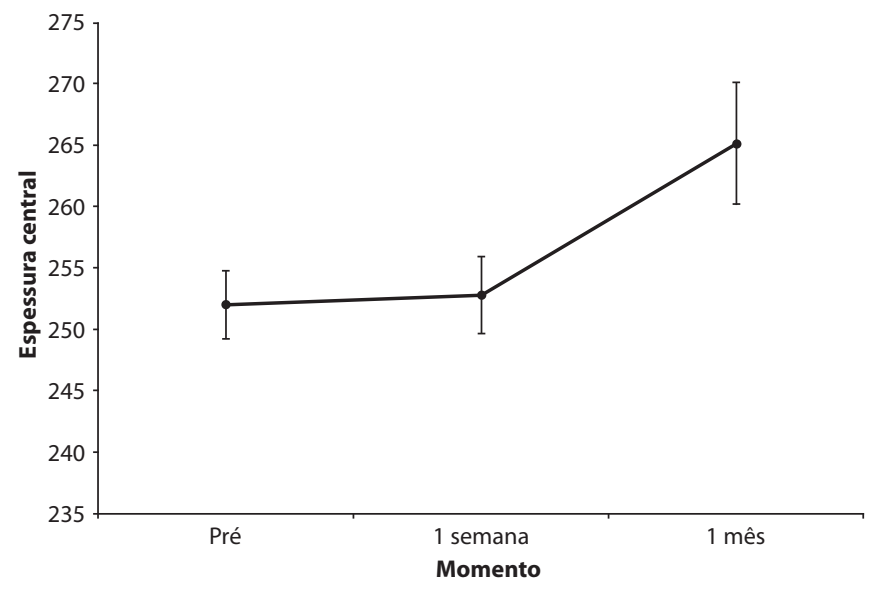

Gráfico 2. Perfil médio da espessura macular central e respectivos erros padrões segundo os momentos.

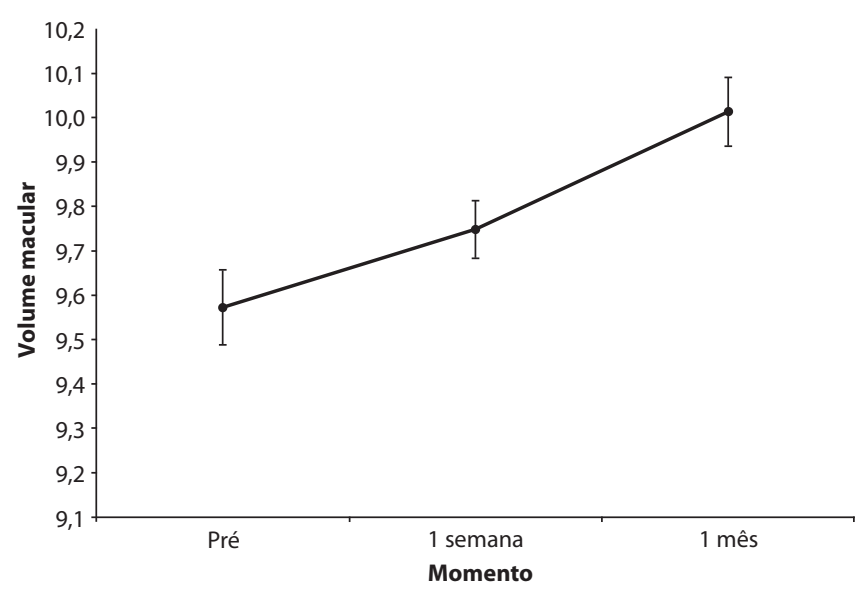

Gráfico 3. Perfil médio do volume macular e respectivos erros padrões segundo os momentos.

dados de 1 mês após a cirurgia, o qual sugere uma relação inversa fraca entre a medida da AV e a espessura central. Parece que quanto maior é o valor da espessura, menor é o valor da medida da AV em logMAR, mostrando mais uma vez que ocorre melhora na acuidade 
Tabela 1. Descrição das medidas avaliadas segundo os momentos e resultado dos testes de comparação entre todos os momentos

\begin{tabular}{|c|c|c|c|c|c|c|c|}
\hline Variável & Média & DP & Mediana & Mínimo & Máximo & $\mathbf{N}$ & $\mathbf{P}$ \\
\hline $\begin{array}{l}\text { Acuidade } \\
\text { visual }\end{array}$ & & & & & & & $<0,001 *$ \\
\hline Pré & 0,52 & 0,27 & 0,40 & 0,20 & 1,30 & 62 & \\
\hline 1 semana & 0,21 & 0,16 & 0,20 & 0,00 & 0,60 & 62 & \\
\hline 1 mês & 0,13 & 0,15 & 0,05 & 0,00 & 0,70 & 62 & \\
\hline $\begin{array}{l}\text { Espessura } \\
\text { central }\end{array}$ & & & & & & & 0,001 \\
\hline Pré & 251,98 & 21,61 & 250,5 & 181 & 305 & 62 & \\
\hline 1 semana & 252,76 & 24,87 & 252,0 & 187 & 332 & 62 & \\
\hline 1 mês & 265,10 & 39,47 & 258,5 & 186 & 492 & 62 & \\
\hline $\begin{array}{l}\text { Espessura } \\
\text { superior }\end{array}$ & & & & & & & $<0,001$ \\
\hline Pré & 312,40 & 16,29 & 313,0 & 255 & 352 & 62 & \\
\hline 1 semana & 313,55 & 17,89 & 314,0 & 262 & 358 & 62 & \\
\hline 1 mês & 322,11 & 22,95 & 318,0 & 272 & 422 & 62 & \\
\hline $\begin{array}{l}\text { Espessura } \\
\text { inferior }\end{array}$ & & & & & & & $<0,001$ \\
\hline Pré & 312,00 & 14,10 & 311,0 & 279 & 349 & 62 & \\
\hline 1 semana & 313,35 & 14,53 & 312,0 & 282 & 352 & 62 & \\
\hline 1 mês & 320,02 & 17,15 & 318,0 & 283 & 378 & 62 & \\
\hline $\begin{array}{l}\text { Espessura } \\
\text { nasal }\end{array}$ & & & & & & & $<0,001$ \\
\hline Pré & 314,79 & 15,18 & 314,0 & 271 & 353 & 62 & \\
\hline 1 semana & 316,50 & 17,59 & 314,5 & 270 & 362 & 62 & \\
\hline 1 mês & 324,27 & 22,27 & 321,5 & 268 & 420 & 62 & \\
\hline $\begin{array}{l}\text { Espessura } \\
\text { temporal }\end{array}$ & & & & & & & $<0,001$ \\
\hline Pré & 301,44 & 16,20 & 302,0 & 255 & 337 & 61 & \\
\hline 1 semana & 303,95 & 14,79 & 304,0 & 268 & 344 & 62 & \\
\hline 1 mês & 311,02 & 18,50 & 310,5 & 275 & 376 & 62 & \\
\hline Volume & & & & & & & $<0,001$ \\
\hline Pré & 9,57 & 0,66 & 9,7 & 8,0 & 10,8 & 61 & \\
\hline 1 semana & 9,75 & 0,51 & 9,7 & 8,5 & 11,2 & 62 & \\
\hline 1 mês & 10,01 & 0,60 & 9,9 & 8,2 & 12,1 & 62 & \\
\hline Inflamação & & & & & & & $<0,001^{*}$ \\
\hline 1 dia & 2,05 & 0,71 & 2 & 1 & 3 & 62 & \\
\hline 1 semana & 0,55 & 0,74 & 0 & 0 & 3 & 62 & \\
\hline 1 mês & 0,13 & 0,53 & 0 & 0 & 3 & 62 & \\
\hline
\end{tabular}

* $=$ resultado do teste de Friedman.

visual apesar de aumentar a espessura macular. Um ponto isolado de grande espessura central corresponde ao caso do paciente com edema macular cistoide. Foi obtido também um diagrama de dispersão entre a inflamação e o volume macular, onde foi incluído apenas os dados de 1 mês após a cirurgia, o qual não parece mostrar nenhuma relação entre estes parâmetros.

A tabela 3 mostra as correlações de Spearman entre a medida da acuidade visual e a espessura central, onde se observa uma relação inversa entre estes parâmetros apenas para 1 semana após a cirurgia $(p<0,05)$, ou seja, quanto menor é o valor da acuidade visual em logMAR (melhor acuidade visual), maiores são os valores da espessura central e do volume para este momento descrito, porém com relação esta-
Tabela 2. Resultado das comparações múltiplas para cada medida entre os momentos dois a dois.

\begin{tabular}{llccc}
\hline Variável & Comparação & $\begin{array}{c}\text { Diferença } \\
\text { média }\end{array}$ & $\begin{array}{c}\text { Erro } \\
\text { padrão }\end{array}$ & P \\
\hline Acuidade visual & & Valor Z & \\
& Pré - 1 semana & 5,34 & $<\mathbf{0 , 0 0 1}$ \\
& Pré - 1 mês & 8,04 & $<\mathbf{0 , 0 0 1}$ \\
& 1 semana - 1 mês & 2,69 & $\mathbf{0 , 0 0 7}$
\end{tabular}

Espessura central

\begin{tabular}{|c|c|c|c|c|}
\hline & Pré - 1 semana & $-0,77$ & 1,70 & 1,000 \\
\hline & Pré - 1 mês & $-13,11$ & 4,08 & 0,006 \\
\hline & 1 semana - 1 mês & $-12,34$ & 3,73 & 0,005 \\
\hline \multicolumn{5}{|c|}{ Espessura superior } \\
\hline & Pré - 1 semana & $-1,15$ & 0,74 & 0,384 \\
\hline & Pré - 1 mês & $-9,71$ & 1,86 & $<0,001$ \\
\hline & 1 semana - 1 mês & $-8,56$ & 1,62 & $<0,001$ \\
\hline \multicolumn{5}{|c|}{ Espessura inferior } \\
\hline & Pré - 1 semana & $-1,35$ & 0,71 & 0,186 \\
\hline & Pré - 1 mês & $-8,02$ & 1,18 & $<0,001$ \\
\hline & 1 semana - 1 mês & $-6,66$ & 0,94 & $<0,001$ \\
\hline \multicolumn{5}{|c|}{ Espessura nasal } \\
\hline & Pré - 1 semana & $-1,71$ & 0,88 & 0,170 \\
\hline & Pré - 1 mês & $-9,48$ & 1,71 & $<0,001$ \\
\hline & 1 semana - 1 mês & $-7,77$ & 1,54 & $<0,001$ \\
\hline \multicolumn{5}{|c|}{ Espessura temporal } \\
\hline & Pré - 1 semana & $-2,61$ & 1,04 & 0,044 \\
\hline & Pré - 1 mês & $-9,75$ & 1,69 & $<0,001$ \\
\hline & 1 semana - 1 mês & $-7,15$ & 1,16 & $<0,001$ \\
\hline \multicolumn{5}{|l|}{ Volume } \\
\hline & Pré - 1 semana & $-0,20$ & 0,06 & 0,006 \\
\hline & Pré - 1 mês & $-0,44$ & 0,08 & $<0,001$ \\
\hline & 1 semana - 1 mês & $-0,25$ & 0,04 & $<0,001$ \\
\hline \multirow[t]{4}{*}{ Inflamação } & & Valor Z & & $\mathbf{P}$ \\
\hline & 1 dia - 1 semana & 6,23 & & $<0,001$ \\
\hline & 1 dia - 1 mês & 8,61 & & $<0,001$ \\
\hline & 1 semana - 1 mês & 2,39 & & 0,017 \\
\hline
\end{tabular}

tística pouco expressiva. Não foram observadas correlações entre a medida da acuidade visual e o volume macular.

Foram realizadas correlações de Spearman entre a inflamação e o volume macular, e entre a inflamação e a espessura central, conforme pode ser observado na tabela 4, a qual mostra que há correlação direta estatisticamente significativa entre a inflamação e o volume macular apenas 1 mês após a cirurgia $(r=0,285$ e $p=0,025)$, ou seja, em 1 mês após a cirurgia, quanto maior o valor da inflamação maior é o volume macular, porém com uma relação estatística fraca. Não foram observadas correlações entre a inflamação e a espessura macular central.

\section{DISCUSSÃO}

No presente estudo foram selecionadas apenas cataratas com baixa densidade, e que permitissem um bom exame pelo OCT, e 


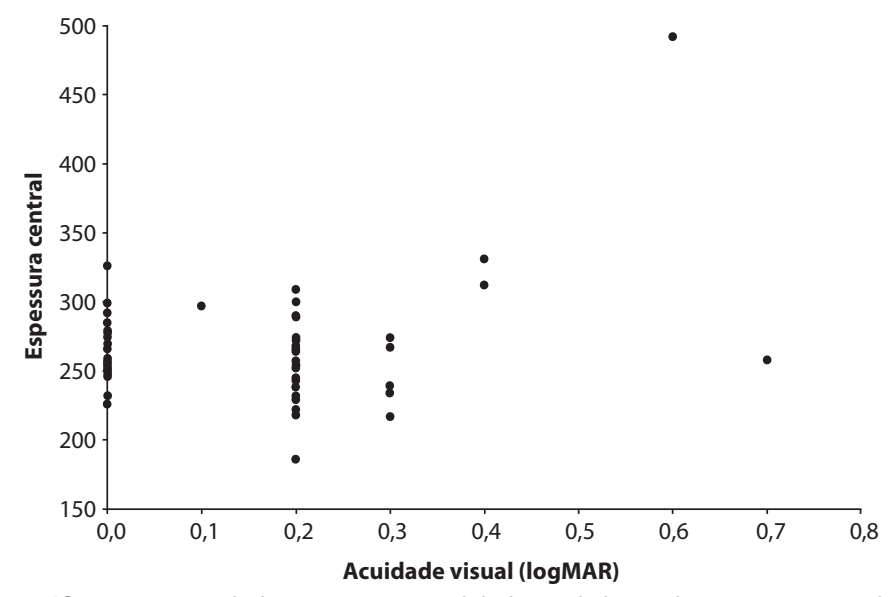

Gráfico 4. Diagrama de dispersão entre a medida da acuidade visual e a espessura central

\begin{tabular}{|c|c|c|c|c|}
\hline Momento & Variáveis & Correlação & $\mathbf{N}$ & $\mathbf{p}$ \\
\hline \multirow[t]{2}{*}{ Pré } & $A \vee x$ espessura central & $-0,065$ & 62 & 0,615 \\
\hline & AV $\times$ volume & $-0,214$ & 61 & 0,097 \\
\hline \multirow[t]{2}{*}{1 semana } & AV x espessura central & $-0,259$ & 62 & 0,042 \\
\hline & AV $\times$ volume & $-0,249$ & 62 & 0,051 \\
\hline \multirow[t]{2}{*}{1 mês } & $A V \times$ espessura central & $-0,031$ & 62 & 0,814 \\
\hline & $A V \times$ volume & 0,006 & 62 & 0,961 \\
\hline
\end{tabular}

Tabela 4. Correlações de Spearman entre a inflamação e a espessura central e volume

\begin{tabular}{lcccc}
\hline Momento & Variáveis & Correlação & N & p \\
\hline 1 dia/pré & Inflamação x espessura central & 0,238 & 62 & 0,063 \\
& Inflamação x volume & $-0,045$ & 61 & 0,732 \\
\multirow{2}{*}{ s semana } & Inflamação x espessura central & 0,068 & 62 & 0,599 \\
& Inflamação x volume & 0,232 & 62 & 0,070 \\
\multirow{2}{*}{ mês } & Inflamação x espessura central & 0,071 & 62 & 0,583 \\
& Inflamação x volume & 0,285 & 62 & $\mathbf{0 , 0 2 5}$ \\
\hline
\end{tabular}

foram descartados todos os exames com nota igual ou inferior a 6, para minimizar o efeito da melhor obtenção das imagens pelo aparelho após a cirurgia de catarata, o que poderia implicar em falsos aumentos da espessura e do volume macular ${ }^{(17)}$.

Foi realizada medida da acuidade visual com a melhor correção antes da cirurgia e após um mês desta, e nas visitas entre estas, foi aferida a acuidade visual com e sem buraco estenopeico, devido à rotina do serviço de realizar refração após um mês da cirurgia. Os dados de acuidade que foram utilizados no presente estudo foram dos resultados prévios à cirurgia e nos dias sete e 30 após esta, portanto, os dados do sétimo dia de operados não foram com a melhor correção, o que deve ser levado em conta ao se analisarem os resultados.

Foram realizadas comparações entre os parâmetros aferidos antes da cirurgia com os obtidos após, utilizando apenas os dados do olho operado, conforme feito nos estudos de alguns autores ${ }^{(18,19)}$, o que permitiu portanto utilizar os dados dos dois olhos de alguns pacientes. Não foi feito controle com o olho contralateral, conforme realizado em outros estudos, o que traria um maior controle dos resultados ${ }^{(17,20)}$.

No presente estudo, foi demonstrado que há aumento da espessura e do volume macular após uma cirurgia não complicada de facoemulsificação com implante de lente intraocular, a partir da primeira semana de pós-operatório em pacientes sem fatores de risco para edema macular. O aumento da espessura parece ocorrer de maneira uniforme, acometendo tanto a região central da mácula como os três milímetros perifoveais nos quatro quadrantes. Em seu estudo, Perente et al. ${ }^{(16)}$, observaram aumento da espessura macular inicialmente nos quadrantes nasal e inferior, o qual se estendeu posteriormente até a fóvea. Alguns autores ${ }^{(20)}$ mediram a espessura foveal e perifoveal de 3 a $6 \mathrm{~mm}$ em 71 olhos, e observaram que há aumento estatisticamente significante na espessura com sete dias após a facoemulsificação, que se tornou maior com um mês de seguimento, e sofreu pequena diminuição com dois meses. Também avaliaram um subgrupo de 34 olhos após seis meses da cirurgia, e observaram um aumento residual na espessura macular estatisticamente não significante $(p>0,05)$, semelhante ao observado por outro autor ${ }^{211}$. Nossos dados indicaram que há um aumento na espessura macular, de menor intensidade e estatisticamente não significante na primeira semana, e que esta se acentua em um mês e se torna significante. Já o volume macular sofre um aumento mais contínuo e progressivo após a cirurgia, sendo estatisticamente significante desde o sétimo dia após a cirurgia. Outro estudo(20) observou edema perifoveal subclínico com OCT dos dias sete a seis meses, e que a medida da espessura macular dos 3 a $6 \mathrm{~mm}$ perifoveais, provê medida mais acurada do edema macular quando comparada com a espessura mínima foveal e a espessura foveal.

Os três mecanismos mais comumente implicados na formação do edema macular pós-operatório são o mecanismo de tração devido à adesão vitreorretiniana e o aumento da produção de prostaglandinas secundária à isquemia do segmento anterior e à liberação de radicais livres devido a maior exposição da retina à luz no período pós-operatório ${ }^{(3,9,16)}$. A constatação de que ocorre edema macular em olhos nos quais não se identifica mecanismos de tração aumenta ainda mais a importância das prostaglandinas e dos radicais livres nesse processo.

As baixas contagens de células na câmara anterior e sua rápida diminuição no período pós-operatório sugerem que ocorre quebra da barreira hematorretiniana, porém de maneira fugaz e limitada na cirurgia de catarata não complicada. Mesmo efêmera essa resposta inflamatória e a alteração da permeabilidade vascular dela decorrente já são suficientes para ocasionar o dano anatômico na retina. Um estudo ${ }^{(22)}$ detectou defeito na barreira hematorretiniana em $97 \%$ dos pacientes após cirurgia de catarata, mesmo nas cirurgias com pequenas incisões. Vale a ressalva de que a medida da inflamação ocular é subjetiva, e que portanto, pode variar entre diferentes examinadores, o que poderia gerar um viés de aferição, mas como foi dito anteriormente, não houve diferença estatisticamente significante entre os resultados dos dois examinadores.

No presente estudo, foi observado apenas edema macular subclínico, ou seja, aquele não observado no exame biomicroscópico de fundo, sendo evidenciado apenas pelo OCT macular. O edema macular também não repercutiu clinicamente, já que praticamente todos os pacientes evoluíram com melhora progressiva da acuidade visual, inclusive no período de maior espessamento retiniano, entre uma semana e um mês de pós-operatório. Inclusive puderam ser observadas poucas correlações inversas fracas entre a medida da acuidade visual e a espessura central e o volume macular, ainda que em apenas alguns momentos, conforme pode ser observado no gráfico 4 e na tabela 3, o que mostra que quanto maior era o volume e a espessura central melhor era a acuidade visual, o que é justamente o contrário do que esperávamos encontrar no inicio do estudo. Estas correlações podem ser explicadas devido à melhora na acuidade visual devido à extração da catarata, e também porque 
não há uma correlação direta entre a espessura e o volume macular com a acuidade visual. Ocorreu apenas um caso de edema macular cistoide em um paciente operado, o qual se manifestou após um mês da cirurgia, com piora da acuidade visual. Neste caso pontual, com o tratamento clinico efetuado e após três meses da cirurgia, a visão se normalizou e a espessura macular encontrava-se praticamente normalizada, porém ainda com edema residual. No estudo de Cagini et al. ${ }^{(18)}$, entre 62 olhos operados, apenas 2 desenvolveram edema macular cistoide, e no estudo de von Jagow et al. ${ }^{(17)}$, não foi observado nenhum caso desta patologia entre 33 olhos operados, o que mostra que este evento é raro após facoemulsificação não complicada. De forma semelhante ao presente estudo, estes dois estudos também observaram um aumento assintomático na espessura e no volume macular após a cirurgia, e que não há correlação entre as alterações maculares e a acuidade visual com a melhor correção (AVMC). Já outro estudo(19) mostrou correlação significante entre a espessura macular mínima foveal e a AVMC no primeiro dia e após 6 semanas da cirurgia, mostrando que nestes momentos os 10 pacientes com maior espessura macular tiveram acuidade visual significativamente mais baixa que os outros pacientes.

Em um estudo ${ }^{(17)}$ foi realizado facoemulsificação com implante de lente intraocular em 33 olhos de 33 pacientes e o olho contralateral serviu como controle, e avaliaram a espessura média mínima foveal (EMMF) e a espessura média foveal (EMF) durante seis semanas após a cirurgia. Encontraram que a EMMF dos olhos operados e a diferença intraindividual da EMMF aumentaram significativamente no primeiro dia, e na sexta semana da cirurgia. A EMF e a diferença intraindividual da EMF aumentaram significativamente no primeiro dia, e na primeira e sexta semanas da cirurgia. A reprodutibilidade foi melhor para EMF do que para EMMF. Não foi encontrada nenhuma correlação entre a espessura macular e a acuidade visual. Parâmetros cirúrgicos e biométricos, como o tempo de facoemulsificação, energia e comprimento axial, não se correlacionaram com o grau de espessamento macular. De forma semelhante ao presente estudo, detectaram um ligeiro aumento na espessura foveal sem impacto sobre a acuidade visual. Este aumento de espessura pode ser devido tanto pelas alterações subclínicas maculares, como pela influência da opacidade média sobre a técnica de medição pelo OCT.

O presente estudo conseguiu demonstrar que há correlação direta fraca estatisticamente significativa entre a inflamação e o volume macular, apenas um mês após a cirurgia, demonstrando que os pacientes que tiveram maior inflamação no pós-operatório, apresentaram também maior volume macular um mês após a cirurgia, ainda que com uma relação estatística fraca. Este efeito pode ser explicado pela quebra da barreira hematorretiniana, o que levaria a um extravasamento vascular, com o consequente aumento do volume macular ${ }^{(22)}$. Não foram observadas correlações entre a inflamação e a espessura macular central.

O OCT possui várias vantagens sobre a angiofluoresceinografia, tais como rapidez, conforto, reprodutibilidade e a não utilização de contraste, além de ser capaz de realizar análises quantitativas ${ }^{(12,13)}$. A nova geração de OCT com tecnologia spectral domain ainda possui propriedades adicionais, com obtenção de imagens de alta resolução, a partir de sinais mais baixos e em alta velocidade ${ }^{(14,15)}$. O presente estudo mostrou que o OCT spectral domain foi capaz de detectar o edema macular subclínico, assim como foi observado também em outros estudos ${ }^{(17-20)}$, e portanto, este parece ser atualmente o melhor método para a detecção desta condição.

A inovação do presente estudo foi a utilização da tecnologia spectral domain na análise do edema macular através do OCT, o que não tinha sido utilizado em nenhum estudo anterior a este com esta finalidade.

A mais importante razão para diminuição da acuidade visual após cirurgia de catarata é o edema macular $r^{(1,2,5)}$ e, por isso, este deve ser melhor estudado e compreendido. Essa alteração deve ser de co- nhecimento do cirurgião, mesmo na ausência de baixa de acuidade visual ou alteração fundoscópica.

\section{CONCLUSÃO}

Edema macular subclínico desenvolve-se mesmo após cirurgia de catarata não complicada em pacientes não predispostos.

O OCT spectral domain foi capaz de detectar pequenos aumentos na espessura macular no período avaliado.

\section{REFERÊNCIAS}

1. Nelson ML, Martidis A. Managing cystoid macular edema after cataract surgery. Curr Opin Ophthalmol. 2003;14(1):39-43.

2. Ray S, D'Amico DJ. Pseudophakic cystoid macular edema. Semin Ophthalmol. 2002; 17(3-4):167-80.

3. Irvine SR. A newly defined vitreous syndrome following cataract surgery. Am J Ophthalmol. 1953;36(5):599-619.

4. Gass JD, Norton EW. Cystoid macular edema and papilledema following cataract extraction: a fluorescein fundoscopic and angiographic study. Arch Ophthalmol. 1966; 76(5):646-61.

5. Mentes J, Erakgun T, Afrash F, Kerci G. Incidence of cystoid macular edema after uncomplicated phacoemulsification. Ophthalmologica 2003;217(6):408-12.

6. Kim SJ, Equi R, Bressler NM. Analysis of macular edema after cataract surgery in patients with diabetes using optical coherence tomography. Ophthalmology. 2007; 114(5):881-9.

7. Ursell PG, Spalton DJ, Whitcup SM, Nussenblatt RB. Cystoid macular edema after phacoemulsification: relationship to blood-aqueous barrier damage and visual acuity. J Cataract Refract Surg. 1999;25(11):1492-7. Comment in: J Cataract Refract Surg. 2000; 26(4):474.

8. Jaffe NS, Clayman HM, Jaffe MS. Cystoid macular edema after intracapsular and extracapsular cataract extraction with and without intraocular lens. Ophthalmology. 1982:89(1):25-9.

9. Ohrolff C, Schalnus R, Rothe R, Spitznas M. Role of the posterior capsule in the aqueous-vitreous barrier in aphakic and pseudophakic eyes. J Cataract Refract Surg. 1990;16(2):198-201.

10. Kim SJ, Belair ML, Bressler NM, Dunn JP, Thorne JE, Kedhar SR, et al. A method of reporting macular edema after cataract surgery using optical coherence tomography. Retina. 2008;28(6):870-6.

11. Mujat M, Chan R, Cense B, Park B, Joo C, Akkin T, et al. Retinal nerve fiber layer thickness map determined from optical coherence tomography images. Opt Express. 2005;13(23):9480-91

12. Leitgeb RA, Hitzenberger CK, Fercher AF, Bajraszewski T. Phase-shifting algorithm to achieve high-speed long-deprh-range probing by frequency-domain optical coherence tomography. Opt Lett. 2003;28(22):2201-3.

13. De Boer JF, Cense B, Park BH, Pierce MC, Tearney GJ, Bouma BE. Improved signalto-noise ratio in spectral-domain compared with time-domain optical coherence tomography. Opt Lett. 2003;28(21):2067-9.

14. Nassif N, Cense B, Park BH, Pierce M, Yun S, Bouma B, et al. In vivo high-resolution video-rate spectral-domain optical coherence tomography of the human retina and optic nerve. Opt Express. 2004;12(3):367-76.

15. Chen TC, Cense B, Pierce MC, Nassif N, Park BH, Yun SH, et al. Spectral domain optica coherence tomography: ultra-high speed, ultra-high resolution ophthalmic imaging. Arch Ophthalmol. 2005;123(12):1715-20.

16. Perente I, Utine CA, Ozturker C, Cakir M, Kaya V, Eren $\mathrm{H}$, et al. Evaluation of macular changes after uncomplicated phacoemulsification surgery by optical coherence tomography. Curr Eye Res. 2007;32(3):241-7.

17. von Jagow B, Ohrloff C, Kohnen T. Macular thickness after uneventful cataract surgery determined by optical coherence tomography. Graefes Arch Clin Exp Ophthalmol. 2007;245(12):1765-71

18. Cagini C, Fiore T, laccheri B, Piccinelli F, Ricci MA, Fruttini D. Macular thickness measured by optical coherence tomography in a healthy population before and after uncomplicated cataract phacoemulsification surgery. Curr Eye Res. 2009;34(12):1036-41.

19. Nicholas S, Riley A, Pater H, Neveldson B, Purdie G, Wells AP. Correlations between optical coherence tomography measurement of macular thickness and visual acuity after cataract extraction. Clin Experiment Ophthalmol. 2006;34(2):124-9;quiz 194 Comment in: Clin Experiment Ophthalmol. 2007;35(1):105; author reply 105-6.

20. Biro Z, Balla Z, Kovacs B. Change of foveal and perifoveal thickness measured by OCT after phacoemulsification and IOL implantation. Eye (Lond). 2008;22(1):8-12.

21. Binder SP. OCT detects higher than expected incidence of macular edema after cataract surgery. Eurotimes. 2004;9(11):1-3.

22. Lobo CL, Faria PM, Soares MA, Bernardes RC, Cunha-Vaz JG. Macular alterations after small-incision cataract surgery. J Cataract Refract Surg. 2004;30(4):752-60. 